\title{
Bone Mesenchymal Stem Cells Contribute to Ligament Regeneration and Graft-Bone Healing after Anterior Cruciate Ligament Reconstruction with Silk-Collagen Scaffold
}

\author{
Fanggang Bi $\mathbb{D}^{1},{ }^{1}$ Yangdi Chen $\left(\mathbb{D},{ }^{2}\right.$ Junqi Liu $\mathbb{D}^{,},{ }^{3}$ Wenhao Hu $\mathbb{D}^{4},{ }^{4}$ and Ke Tian $\mathbb{D}^{1}$ \\ ${ }^{1}$ Department of Orthopedic Surgery, The First Affiliated Hospital of Zhengzhou University, No. 1 Jianshe East Road, Zhengzhou, \\ China 450001 \\ ${ }^{2}$ Henan University of Chinese Medicine, No. 156 Jinshui East Road, Zhengzhou, China 450001 \\ ${ }^{3}$ Department of Radiation Oncology, The First Affiliated Hospital of Zhengzhou University, No. 1 Jianshe East Road, Zhengzhou, \\ China 450001 \\ ${ }^{4}$ Spine Division, Department of Orthopedics, The Fourth Medical Center of PLA General Hospital, No. 51 Fucheng Road, \\ Beijing, China
}

Correspondence should be addressed to Fanggang Bi; 163bfg@163.com

Received 10 November 2020; Revised 25 January 2021; Accepted 15 April 2021; Published 24 April 2021

Academic Editor: Gianpaolo Papaccio

Copyright (c) 2021 Fanggang Bi et al. This is an open access article distributed under the Creative Commons Attribution License, which permits unrestricted use, distribution, and reproduction in any medium, provided the original work is properly cited.

\begin{abstract}
Anterior cruciate ligament (ACL) reconstruction was realized using a combination of bone mesenchymal stem cells (BMSCs) and silk-collagen scaffold, and an in vivo evaluation of this combination was performed. By combining type I collagen and degummed silk fibroin mesh, silk-collagen scaffolds were prepared to simulate ligament components. BMSCs isolated from bone marrow of rabbits were cultured for a homogenous population and seeded on the silk-collagen scaffold. In the scaffold and BMSC (S/C) group, scaffolds were seeded with BMSCs for $72 \mathrm{~h}$ and then rolled and used to replace the ACL in 20 rabbits. In the scaffold (S) group, scaffolds immersed only in culture medium for $72 \mathrm{~h}$ were used for ACL reconstruction. Specimens were collected at 4 and 16 weeks postoperatively to assess ligament regeneration and bone integration. HE and immunohistochemical staining (IHC) were performed to assess ligament regeneration in the knee cavity. To assess bone integration at the graft-bone interface, HE, Russell-Movat staining, micro-CT, and biomechanical tests were performed. After 4 weeks, vigorous cell proliferation was observed in the core part of the scaffold in the S/C group, and a quantity of fibroblast-like cells and extracellular matrix (ECM) was observed in the center part of the graft at 16 weeks after surgery. At 4 and 16 weeks postoperatively, the tenascin-C expression in the $\mathrm{S} / \mathrm{C}$ group was considerably higher than that in the $\mathrm{S}$ group $(4 \mathrm{w}, p<0.01 ; 16 \mathrm{w}, p<0.01)$. Furthermore, bone integration was better in the S/C group than in the S group, with histological observation of trabecular bone growth into the graft and more mineralized tissue formation detected by micro-CT ( $4 \mathrm{w}$, bone volume fraction (BV/TV), $p=0.0169$, bone mineral density (BMD), $p=0.0001 ; 16 \mathrm{w}, \mathrm{BV} / \mathrm{TV}, p=0.1233$, BMD, $p=0.0494$ ). These results indicate that BMSCs promote ligament regeneration in the knee cavity and bone integration at the graft-bone interface. Silk-collagen scaffolds and BMSCs will likely be combined for clinical practice in the future.
\end{abstract}

\section{Introduction}

The anterior cruciate ligament (ACL) is a main structure that maintains stability of the knee [1]. As a common athletic injury, ACL rupture can cause serious damage such as knee joint instability, injury to other ligaments, dislocation, and osteoarthritis $[2,3]$. ACL reconstruction is currently consid- ered the gold standard for treating ACL rupture, and grafts including autografts, allografts, and synthetic grafts are used for this purpose [4-6]. However, these grafts have some limitations. Shortcomings of autografts include long surgery time, donor site complications, long rehabilitation time, and decrease in knee range of motion [7]. Disadvantages of allografts include higher cost, higher infection rates, and a 
higher failure rate compared to autografts [8]. The complications of permanent synthetic grafts include osteoarthritis, chronic synovitis, foreign-body response, and long-term rupture [9]. Bone mesenchymal stem cells (BMSCs) and ligament tissue engineering have become promising techniques for addressing these drawbacks.

To reconstruct the ACL well, tissue engineering needs to meet the following criteria: provide immediate joint stability after surgery, assure good ligament regeneration in the knee cavity as the engineered tissue gradually degrades and diminishes, and establish good bone integration at the graft-bone interface for long-term stability after surgery [10]. In a previous study, we designed a graft by combining collagen matrix with knitted degummed silk fibroin to reconstruct the ACL in a rabbit model [11]. The silk-collagen scaffold was discovered to have good biocompatibility and biomechanical properties [12]. However, in the early postoperative period, limited ingrowth of the newly regenerated connective tissue in the knee cavity restricted ligament regeneration, and bone tissue in the bone tunnel disrupted graft-bone healing [13, 14].

BMSCs are pluripotent cells and have become a very important source of cells for cell therapy and engineered tissue repair [15]. Their multiple differentiation potential for therapeutic application when implanted with biodegradable scaffolds has been demonstrated in several previous studies [16-19]. Although which cell types initiate and regulate the ligament regeneration and graft-bone healing process has not been clarified [20], it seems that BMSCs in the marrow from the bone tunnel promote ligament regeneration and repair at the graft-bone interface [21]. Lim et al. demonstrated that the failure load and stiffness of MSC-enhanced hamstring tendons were obviously greater at 8 weeks after ACL reconstruction surgery in a rabbit model [22]. According to Soon et al., MSCs may form an intermediate fibrocartilage zone between bone and the allograft tendon after reconstruction surgery [23].

No unified and widely accepted approach has been available to guide how BMSCs are applied. BMSC application approaches include local injection [24], BMSCs sheet technology [25], combination with fibrin glue or collagen gel $[17,26]$, and implantation on a scaffold for tissue engineering $[27,28]$. Implanting BMSCs on a tissue-engineering scaffold seems more reliable because of its small distraction on the growing status and environment of BMSCs. Based on this background, we seeded BMSCs on a silk-collagen scaffold, attempting to determine whether BMSCs could promote ligament regeneration in the knee cavity and graft-bone healing. In the present study, a rabbit ACL reconstruction model with silk-collagen scaffold with or without BMSCs was established. We hypothesized that BMSCs could improve knee ligament regeneration and bone integration at the graft-bone interface, as demonstrated by histological assessment, micro-CT, and a biomechanical test.

\section{Materials and Methods}

2.1. Scaffold Preparation. The raw silk fibers were provided by Zhejiang Cathaya International, Ltd. The degumming pro- cess was completed using $0.02 \mathrm{M} \mathrm{Na} \mathrm{Na}_{3} \quad\left(100^{\circ} \mathrm{C}\right.$ for $60 \mathrm{~min}, 3$ times) to extract sericin, as described in a previous study [11]. Isolation and purification of the collagen matrix from pigs' Achilles tendons were performed with dilute acid and neutral salt extractions [29]. The knitted silk mesh extracted from sericin was soaked in acidic collagen solution (type I, pH 3.2, w/v $1 \%)$, freeze-dried $\left(-80^{\circ} \mathrm{C}\right.$ for $12 \mathrm{~h}$ followed by Heto PowerDry LL1500 for $24 \mathrm{~h}$ ), and subjected to dehydrothermal crosslinking in a vacuum oven (30 mTorr, $105^{\circ} \mathrm{C}$ for $24 \mathrm{~h}$ ) [11]. Observation of the surface microstructures of the raw silk, degummed silk, and silk-collagen scaffold was performed using a scanning electron microscope (SEM). Finally, cobalt-60-sterilized silk-collagen scaffolds were prepared for the following evaluations.

2.2. Isolation, Culture, and Identification of BMSCs. Bone marrow aspirates extracted from New Zealand White rabbits $(2.5 \pm 0.2 \mathrm{~kg}, 12$ weeks old) were used to isolate and culture BMSCs, as previously described [17]. Mononuclear cells were gathered in Ficoll-Hypaque gradient (Sigma) after centrifugation and then suspended in cell culture medium containing $10 \%$ fetal bovine serum (FBS, Gibco). As the culture medium changed, the suspended cells were removed after culture at $37^{\circ} \mathrm{C}$ in $5 \% \mathrm{CO}_{2}$ for $72 \mathrm{~h}$. When the adherent cells reached $70-80 \%$ confluence, subculture was performed. After culture for 2 weeks, a homogenous BMSC population was obtained, and the third passage was collected and seeded on the silkcollagen scaffold. BMSCs adherent on the scaffold were observed by SEM after being seeded for $72 \mathrm{~h}$. The osteogenic, adipogenic, and chondrogenic differentiation abilities of passage 3 cells were identified after culture with special inducing media (Gibco) for 3 weeks. Finally, alizarin red (Sangon), oil red O (Sangon), and alcian blue staining (Sangon) were performed according to the manufacturer's protocols.

2.3. Flow Cytometry. To confirm the homogeneous property of passage 3 BMSCs cultured at 2 weeks, a characterization for stemness markers was performed. Sheep anti-rabbit antibodies of CD29, CD73, CD105, and phycoerythrin-(PE-) labeled IgG secondary antibody were purchased from the eBioscience (San Diego, CA). Approximately $3 \times 10^{5}$ cells were harvested and resuspended in $100 \mu \mathrm{L}$ phosphatebuffered saline (PBS). Cells were incubated with primary antibodies of CD29 (1:100), CD73 (1:100), and CD105 $(1: 100)$ for 1 hour at $4^{\circ} \mathrm{C}$. Subsequently, the cells were washed with PBS for 3 times, and the supernatant was discarded by centrifugation at $250 \times \mathrm{g}$ for 5 minutes. And the cells were resuspended in $100 \mu \mathrm{L}$ PBS and incubated with PE-labeled secondary sheep antibody $(1: 200)$ for 40 minutes at $4^{\circ} \mathrm{C}$ in the dark. The cells were washed with PBS for 3 times, and the supernatant was discarded by centrifugation at $250 \times \mathrm{g}$ for 5 minutes. The cells were immediately tested on the machine (BD LSRFortessa) after resuspended with $400 \mu \mathrm{L}$ PBS. The data were analyzed with FlowJo 10.0 software.

2.4. Animal Model Study Design. The present study used 40 male New Zealand white rabbits provided by Hualan Biology (2.5-3.0 kg, 12 weeks old, certification No.: 


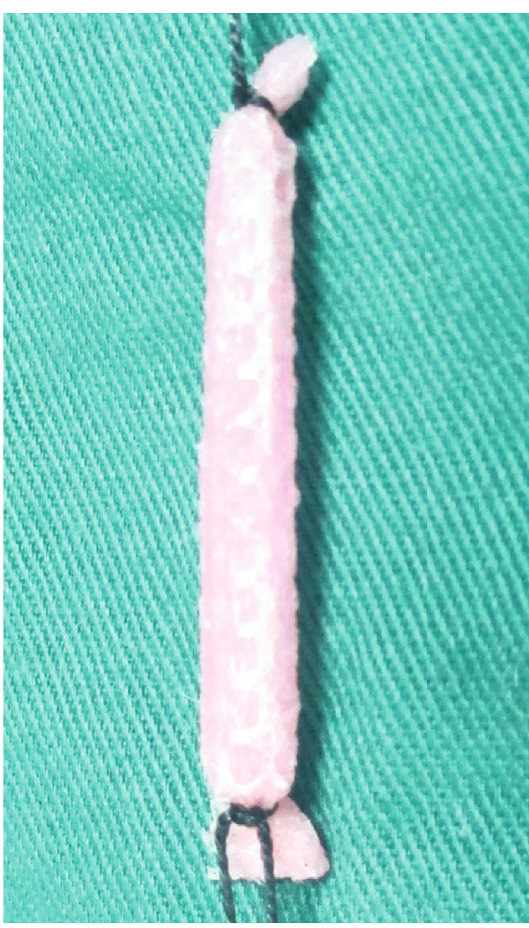

(a)

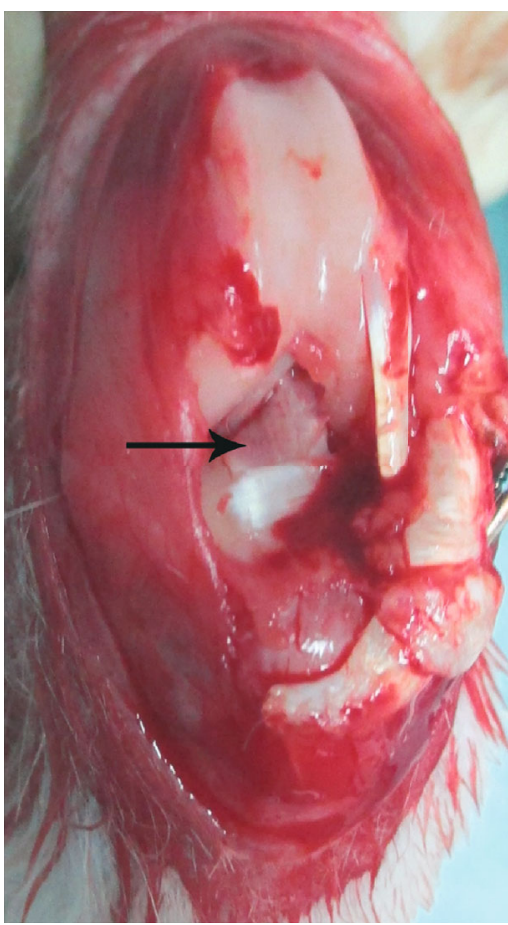

(b)

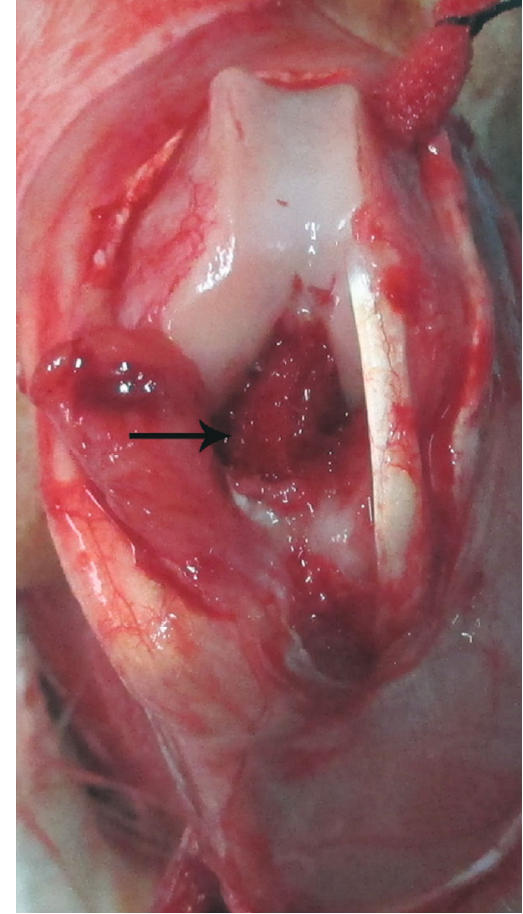

(c)

Figure 1: (a) After coculture with BMSCs in the S/C group and immersion only in culture medium in the S group for $72 \mathrm{~h}$, the scaffold was rolled for use as a graft to replace the native ACL in a rabbit model. (b) General observation of the native ACL: the arrow points to the native ACL. (c) General observation of the knee after ACL reconstruction with the scaffold: the arrow points to the implanted graft.

SYDW20190409). The ethics committee of the First Affiliated Hospital of Zhengzhou University approved the experimental protocol (ethics review No.: 2020-KY-012). Two equal-numbered groups (scaffold group, S; scaffold and BMSCs group, S/C) were formed by dividing the rabbits at random, and ACL reconstruction was carried out in the knee of the left hind leg. In the $S$ group, silk scaffolds were immersed in culture medium for $72 \mathrm{~h}$, whereas in the S/C group, silk scaffolds were seeded with BMSCs for $72 \mathrm{~h}$; then both types of scaffold were rolled and used for ACL reconstruction (Figure 1(a)). At 4 and 16 weeks after the operation, 10 rabbits from each group were sacrificed. Five specimens in each group were assessed for ligament regeneration by hematoxylin and eosin (HE) staining and immunohistochemical (IHC) staining and for bone integration at the graft-bone interface by $\mathrm{HE}$ and RussellMovat (RM) staining. Graft-bone healing was assessed in the remaining specimens $(n=5)$ using micro-CT and the biomechanical test.

2.5. Surgical Procedure. ACL reconstruction was carried out under strict aseptic conditions, and all operations were performed by one person (Bi). After general anesthesia was achieved by pentobarbital (Kyoritsu-seiyaku, $30 \mathrm{mg} / \mathrm{kg}$ body weight), the surgical area was shaved, disinfected, and covered. Exposure of the knee cavity was achieved by a $3 \mathrm{~cm}$ incision along the patellar tendon, and then the native ACL was removed (Figure 1(b)). A $2.0 \mathrm{~mm}$ Kirschner wire was used to make tunnels in the femur and tibia. The graft was inserted through the bone tunnels, and its ends were attached to the surrounding soft tissue and the periosteum with 1-0 Ethibond suture (Figure 1(c)). Then, the rabbits were raised in their cages without restriction after surgery.

2.6. Ligament Regeneration Assessment. After collection, the tibia-graft-femur complexes $(n=5$ per group at each point in time) were immediately put in paraformaldehyde (4\%; Sangon) for $24 \mathrm{~h}$. The graft in the knee cavity was collected, dehydrated, and embedded. After sectioning, the slices were stained with $\mathrm{HE}$. Ligament regeneration was analyzed by immunohistochemistry staining for tenascin-C. Image-Pro Plus 6.0 software (IPP6.0) was used to calculate the average immunoreactivity density of tenacin-C in the graft.

2.7. Graft-Bone Healing Assessment. After the graft was dissected from the tibia-graft-femur complex, bone integration at the graft-bone interface was assessed using the remaining bone samples. The bone samples were decalcified by ethylenediaminetetraacetic acid (EDTA; 10\%) until they could be easily sectioned with a blade. The samples were sectioned after dehydration and embedding, and the slices were stained with $\mathrm{HE}$ and RM to evaluate bone integration.

2.8. Micro-CT Evaluations. The tibia-graft-femur complexes ( $n=5$ per group at each point in time) were prepared for micro-CT scan (36 $\mu \mathrm{m}$ thickness; Skyscan 1176, Bruker, Antwerp, Belgium) and immediately stored at $-80^{\circ} \mathrm{C}$ after collection. The specimens were placed in a refrigerator $\left(4^{\circ} \mathrm{C}\right)$ overnight to thaw before testing. Detection of mineralized tissue regeneration at the graft-bone interface was 


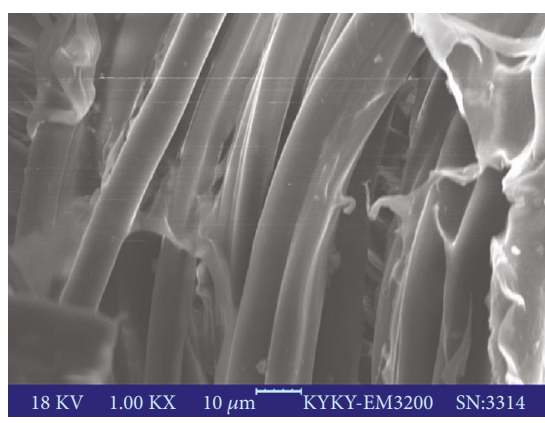

(a)

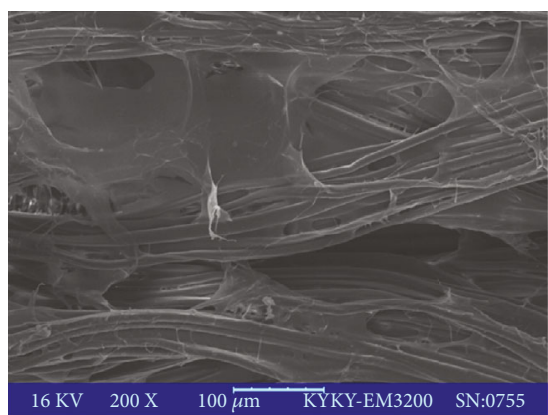

(c)

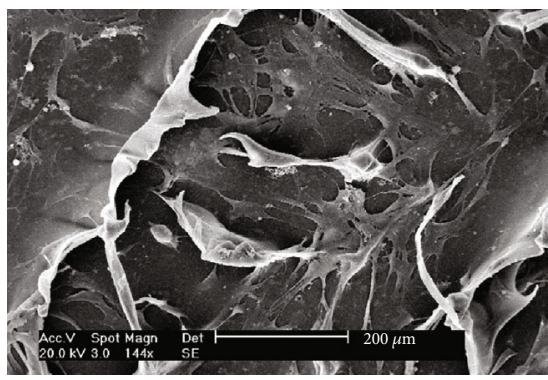

(e)

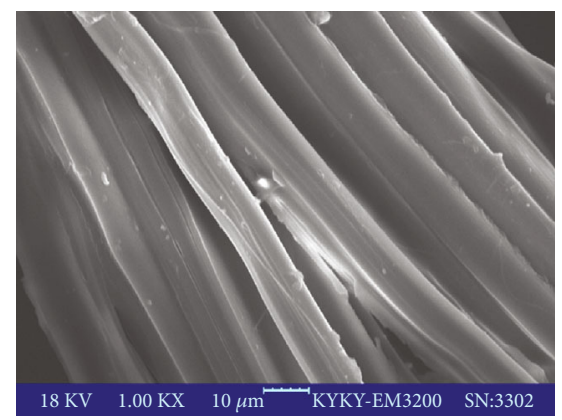

(b)

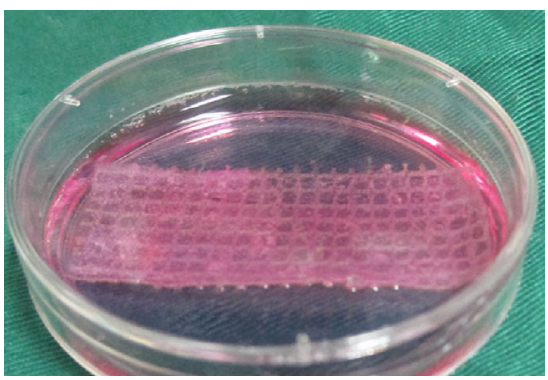

(d)

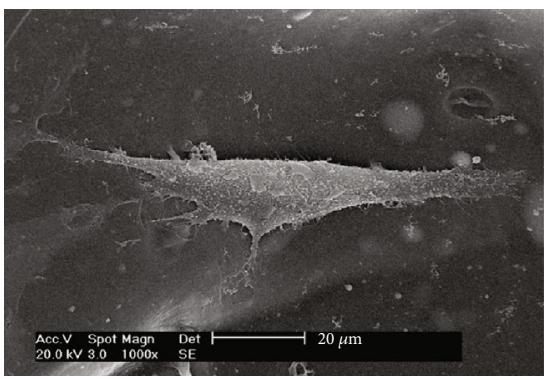

(f)

FIGURE 2: (a) The microstructure of silk fibers before degumming: the surface is coated with the glue-like protein sericin. (b) Under SEM, the surface of silk fibroins becomes smooth after complete degumming; the average diameter is about $10 \mu \mathrm{m}$. (c) The microstructure of silkcollagen scaffold after the dehydrothermal crosslinking process: the collagen sponge permeated into the rings of knitted mesh. (d) BMSCs were seeded on the scaffold and cocultured for $72 \mathrm{~h}$ for further use. (e) BMSCs exhibited vigorous proliferation on the scaffold. (f) BMSCs retained good cellular morphology on the scaffold observed by SEM.

performed using radiograph images. Calculation of the bone mineral density (BMD), trabecular number (Tb.N), bone volume fraction (BV/TV), trabecular thickness (Tb.Th), and trabecular separation (Tb.Sp) of a $2.0 \mathrm{~mm}$ diameter cylinder scope including the graft-bone interface was carried out by 3-dimensional standard microstructural analyses [30].

2.9. Biomechanical Test. The next step was to carry out the biomechanical test. The tibia-graft-femur complex $(n=5$ per group at each point in time) was created by dissecting all soft tissue around the knee joint except for the graft. The femur and tibia were screwed into custom-made steel pipes, and the steel pipes were secured to an Instron 553A biomechanical testing system (Instron). The crosshead speed of the tensile load during the biomechanical test was $5 \mathrm{~mm} / \mathrm{min}$. The elongation $(\mathrm{mm})$ and failure load $(\mathrm{N})$ were documented, and the slope of the recorded curve indicated stiffness
$(\mathrm{N} / \mathrm{mm})$. The tibia-graft-femur complexes were kept moist with normal saline.

2.10. Statistical Analyses. The data collected in the present study are expressed as mean \pm standard deviation (SD). SPSS 16.0 software was used for the statistical analyses. Differences were considered statistically significant at $p<0.05$. Independent-sample $t$-tests were used to detect differences between groups.

\section{Results}

3.1. SEM Observation. The surface of raw silk fibers was irregular due to the sericin coating on the silk fibroin (Figure 2(a)). The silk fibroins, which were about $10 \mu \mathrm{m}$ in diameter and had a smooth surface, were visible after complete degumming (Figure 2(b)). After the process of freezedrying and dehydrothermal crosslinking, the collagen sponge 


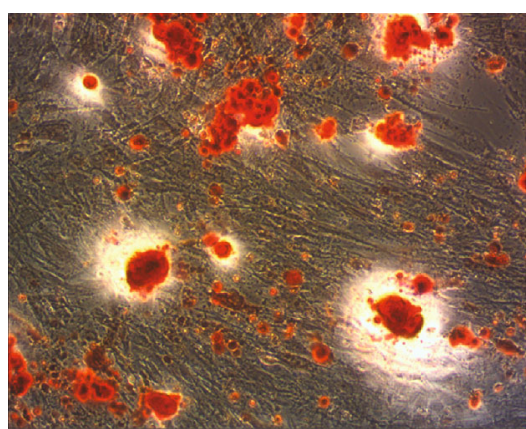

(a)

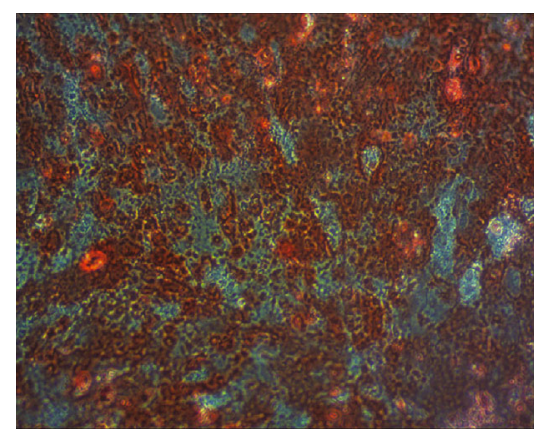

(b)

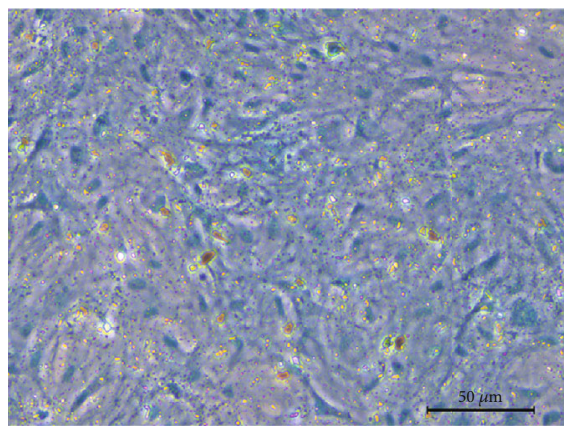

(c)

FIGURE 3: Representative images from alizarin red (a), oil red O (b), and alcian blue (c) staining to detect the osteogenic, adipogenic, and chondrogenic differentiation abilities of passage 3 cells.
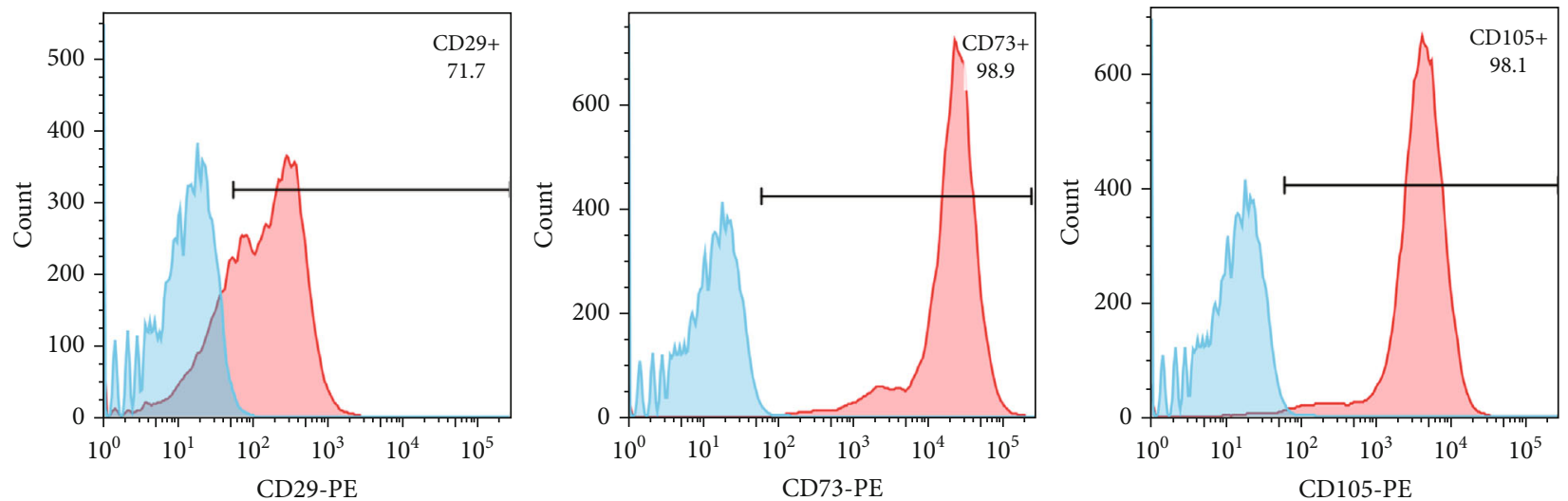

Figure 4: The third passage cells had high expression of CD29 (71.7\%), CD73 (98.9\%), and CD105 (98.1\%).

distributed on the silk fibroin surface entered into the rings of knitted mesh, resulting in a fuzzier surface (Figure 2(c)). BMSCs adhered to the collagen surface after being seeded onto the scaffold for $72 \mathrm{~h}$ in Petri dishes (Figure 2(d)) and maintained good cellular morphology (Figures 2(e) and 2(f)).

3.2. Identification of BMSCs. Alizarin red, oil red $\mathrm{O}$, and alcian blue staining were performed after cells were cultured in the inducing medium for 3 weeks. Mineralized nodules, lipid droplets, and green cytoplasm were observed under the microscope after staining with alizarin red, oil red $\mathrm{O}$, and alcian blue (Figure 3). The results of flow cytometry showed that the third passage cells had high expression of
CD29 (71.7\%), CD73 (98.9\%), and CD105 (98.1\%) (Figure 4).

3.3. Ligament Regeneration Assessment. Cellular infiltration and tenascin- $\mathrm{C}$ production were evaluated by $\mathrm{HE}$ and immunohistochemical staining. In the $\mathrm{S} / \mathrm{C}$ group, considerable cells were observed in the core part of the graft, whereas in the $S$ group, only a few cells could be observed in the graft at 4 weeks postoperatively (Figures 5(a) and 5(b)). At 16 weeks after surgery, fibroblast-like cells became more regular and denser in the $\mathrm{S} / \mathrm{C}$ than in the $\mathrm{S}$ group (Figures 5(c) and $5(d))$. The tenascin- $C$ expression in the $S$ group was obviously lower than that in the S/C group at 4 and 16 weeks after surgery (Figures 6(a) and 6(b)). 


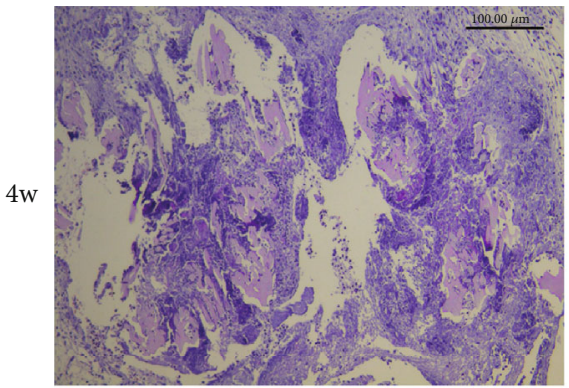

(a)

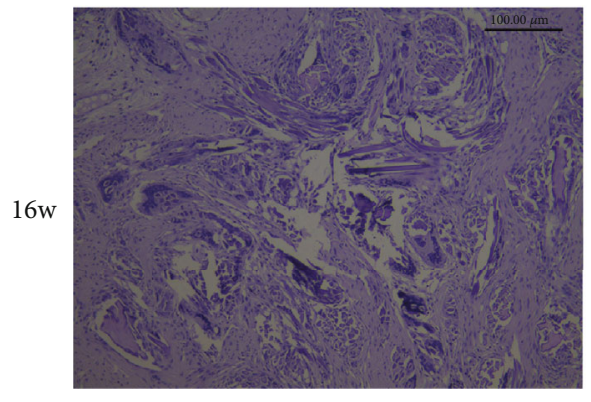

(c)
$\mathrm{S} / \mathrm{C}$

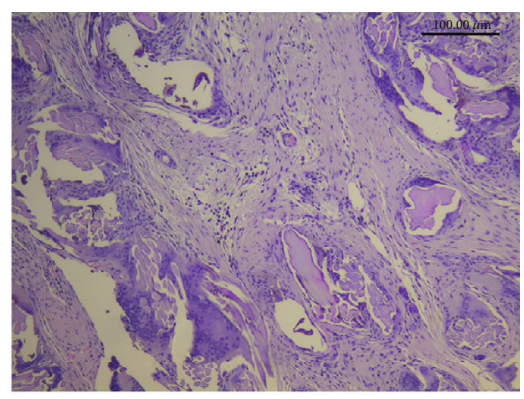

(b)

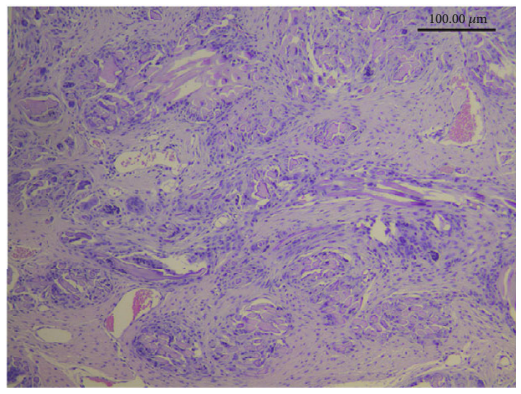

(d)

FiguRE 5: At 4 weeks after surgery, HE staining of grafts in the knee cavity in the $S$ group (a) revealed few cells, whereas considerable cells were observed in the core part of the graft in the S/C group (b). At 16 weeks after surgery, fibroblast-like cells became more regular and denser in the $\mathrm{S} / \mathrm{C}$ group (d) than in the $\mathrm{S}$ group (c).
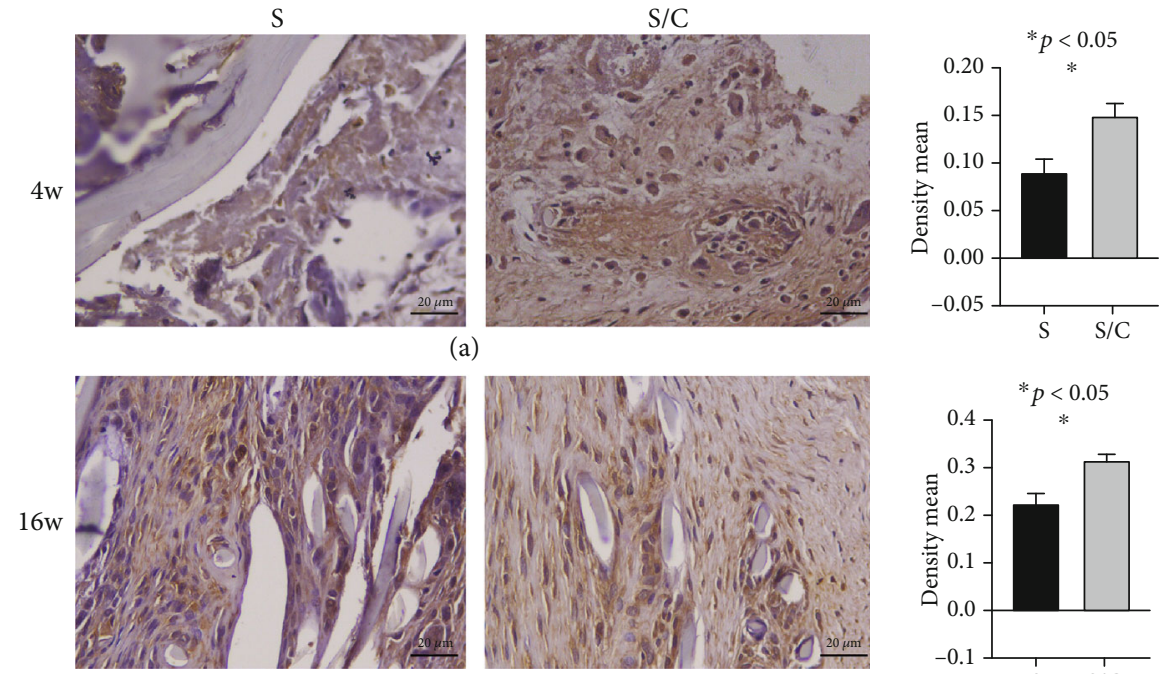

(b)

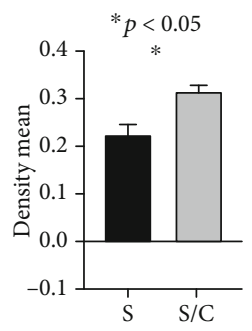

FIGURE 6: Immunohistochemistry staining of grafts in the knee cavity specific for tenascin-C in the S group and S/C group to assess ligament regeneration: the density mean of immunoreactivity was higher in the S/C group than in the S group at 4 weeks (a) and 16 weeks (b) after the operation.

3.4. Graft-Bone Healing Assessment. Histological staining revealed connective tissue with a thin chondrocyte layer at the graft-bone interface at 4 weeks after the reconstruction surgery. No obvious bone integration was noticed in the two groups; although, more cells were distributed in the core part in the S/C group than in the S group (Figures $7(\mathrm{a})$ and $7(\mathrm{~b})$; Figure $8(\mathrm{a})$ and $8(\mathrm{~b}))$. By 16 weeks after surgery, the mature trabecular bone and considerable cell invasion in the scaffold could be noticed at the graft-bone interface in the $S$ group, while integration with the trabecular bone in the graft was observed in the S/C group (Figures 7 (c) and $7(\mathrm{~d})$; Figures $8(\mathrm{c})$ and $8(\mathrm{~d})$ ).

3.5. Micro-CT Evaluations. Micro-CT reconstructed the high-resolution transverse sectional images of the tibia and femur. The formation of the mineralized tissue at the graft- 


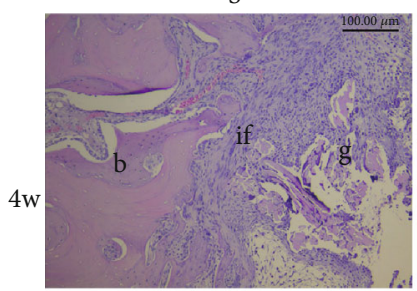

(a)

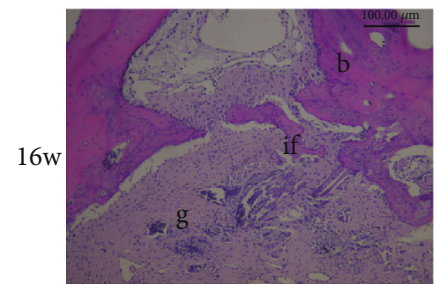

(c)
$\mathrm{S} / \mathrm{C}$

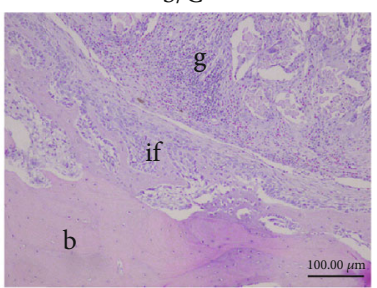

(b)

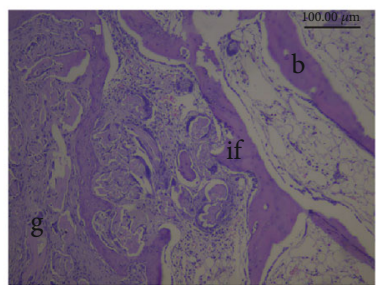

(d)
FIGURE 7: HE staining of the graft-bone interface for histological observation. At 4 weeks after the reconstruction surgery, no obvious bone integration was noticed in the two groups; although, more cells were distributed in the core part in the S/C group (b) than in the S group (a). At week 16, mature trabecular bone and considerable cell invasion in the scaffold could be noticed at the graft-bone interface in the $S$ group (c). In the $S / C$ group, integration of the trabecular bone into the graft was observed $(\mathrm{d})$. g: graft; b: bone; if: interface.

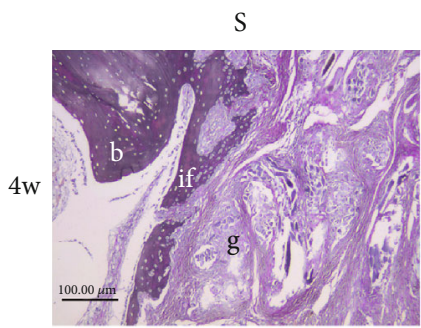

(a)

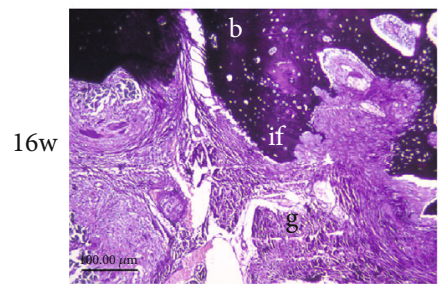

(c)

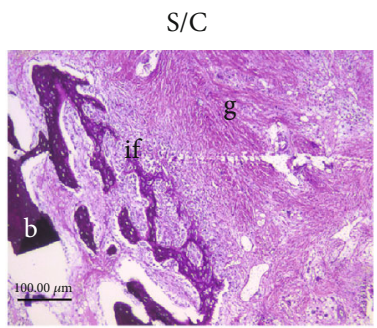

(b)

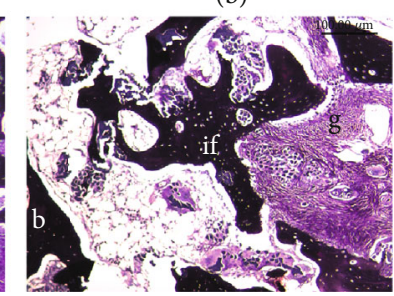

(d)
Figure 8: Russell-Movat staining of the graft-bone interface. At 4 weeks after the reconstruction surgery, no obvious bone integration was noticed in the two groups $(\mathrm{a}, \mathrm{b})$. At week 16, the mature trabecular bone could be noticed at the graft-bone interface in the $S$ group (c), whereas in the $S / C$ group, osteointegration with the trabecular bone into the graft was observed (d). g: graft; b: bone; if: interface.

bone interface could be easily observed. In both groups, few mineralized tissues were detected at the graft-bone interface at 4 weeks postoperatively (Figures 9(a) and 9(b)). BV/TV, $\mathrm{Tb}$. Th, and BMD values were increased significantly more in the $\mathrm{S} / \mathrm{C}$ than in the $\mathrm{S}$ group (Table 1). However, at 16

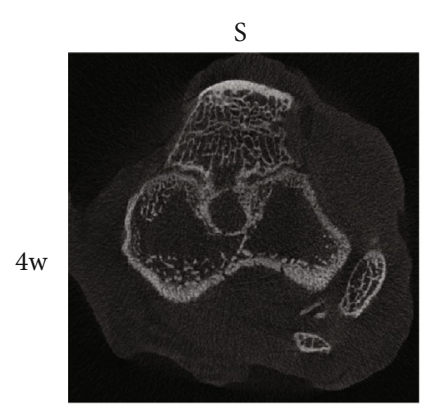

(a)

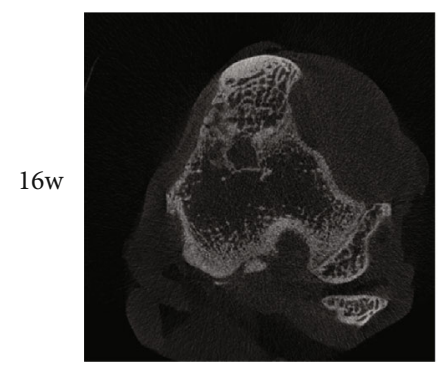

(c)

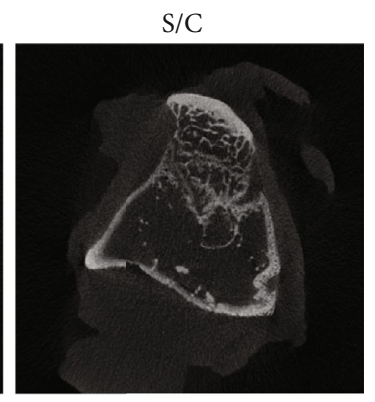

(b)

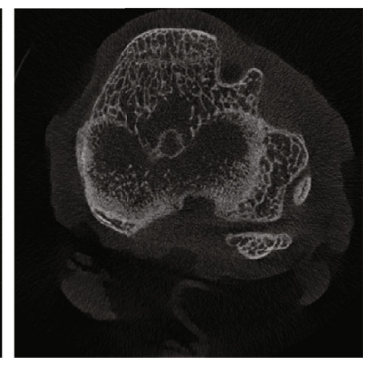

(d)
FIgURE 9: Representative micro-CT images. Few mineralized tissues were detected at the graft-bone interface at 4 weeks postoperatively (a, S group; b, S/C group). At 16 weeks postoperatively, distinct signals appeared indicating new mineralized tissue regeneration at the graft-bone interface of each group (c, S group; d, S/C group). The mineralized tissue signal and average bone tunnel area may indicate bone integration at the graft-bone interface.

TABle 1: Micro-CT evaluations (mean \pm SD). BV/TV, Tb.Th, and BMD increased significantly more in the S/C group than in the $S$ group at 4 weeks after reconstruction surgery. Significant increases in $\mathrm{Tb}$.Th and BMD were observed in the $\mathrm{S} / \mathrm{C}$ group relative to the $\mathrm{S}$ group. ${ }^{*}$ indicates a notable distinction between the two comparison groups.

\begin{tabular}{lcccc}
\hline Time point & Items & S & S/C & $p$ value \\
\hline \multirow{4}{*}{$4 \mathrm{w}$} & BV/TV $(\%)$ & $14.04 \pm 0.41$ & $15.53 \pm 0.90$ & $0.0169^{*}$ \\
& Tb.Th $(\mathrm{mm})$ & $0.27 \pm 0.02$ & $0.32 \pm 0.02$ & $0.0198^{*}$ \\
& Tb.N $(1 / \mathrm{mm})$ & $0.24 \pm 0.07$ & $0.30 \pm 0.01$ & 0.0883 \\
& Tb.Sp $(\mathrm{mm})$ & $1.21 \pm 0.01$ & $1.13 \pm 0.07$ & $0.0426^{*}$ \\
& BMD $\left(\mathrm{mg} / \mathrm{cm}^{3}\right)$ & $0.08 \pm 0.01$ & $0.10 \pm 0.00$ & $0.0001^{*}$ \\
\hline \multirow{4}{*}{$16 \mathrm{w}$} & BV/TV $(\%)$ & $22.29 \pm 1.01$ & $23.50 \pm 0.98$ & 0.1233 \\
& Tb.Th (mm) & $0.29 \pm 0.05$ & $0.36 \pm 0.03$ & $0.0406^{*}$ \\
& Tb.N $(1 / \mathrm{mm})$ & $0.39 \pm 0.10$ & $0.47 \pm 0.07$ & 0.2725 \\
& Tb.Sp (mm) & $0.91 \pm 0.03$ & $0.83 \pm 0.07$ & 0.0887 \\
& BMD $\left(\mathrm{mg} / \mathrm{cm}^{3}\right)$ & $0.19 \pm 0.02$ & $0.22 \pm 0.02$ & $0.0494^{*}$ \\
\hline
\end{tabular}

weeks after reconstruction surgery, distinct signals appeared indicating new mineralized tissue regeneration at the graftbone interface in both groups (Figures 9(c) and 9(d)), with greater increases in $\mathrm{Tb}$. Th and BMD observed in the $\mathrm{S} / \mathrm{C}$ than in the $\mathrm{S}$ group (Table 1).

3.6. Biomechanical Test. All grafts in both groups failed through rupture in the knee cavity or pullout from the bone 


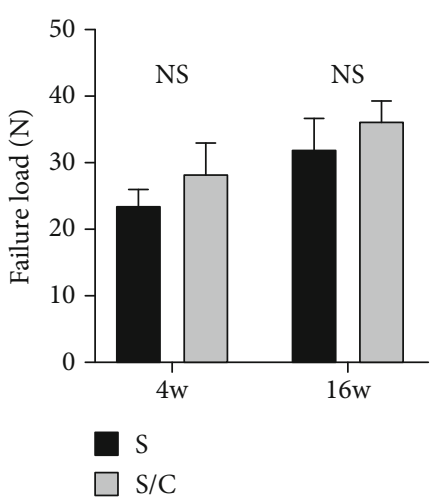

(a)

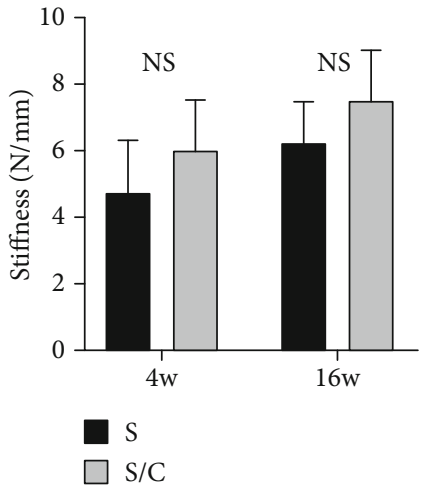

(b)

Figure 10: No significant differences were found in failure load (a) or stiffness (b) between the S group and S/C group at 4 and 16 weeks after the reconstruction surgery. NS indicates no significant difference between groups.

tunnel. No obvious differences in the failure load were found between the two groups at 4 and 16 weeks after surgery $(4 \mathrm{w}$, S $23.24 \pm 2.18$ vs. S/C $28.38 \pm 4.07, p=0.06 ; 16 \mathrm{w}, \mathrm{S} 31.85$ \pm 4.24 vs. $36.36 \pm 2.58, p=0.11$; Figure 10 (a)). Stiffness was calculated by recording the displacement and failure load from the load-deformation curve. The stiffness was not considerably different between the groups at 4 and 16 weeks after surgery ( $4 \mathrm{w}, \mathrm{S} 4.71 \pm 1.42$ vs. S/C $4.71 \pm 1.42, p=0.21 ; 16 \mathrm{w}$, S $6.18 \pm 1.17$ vs. $7.52 \pm 1.31, p=0.16$; Figure $10(\mathrm{~b}))$.

\section{Discussion}

Tissue engineering grafts for ACL reconstruction have focused on ligament regeneration in the knee cavity and bone integration at the graft-bone interface [31]. The present study revealed that BMSCs promoted ligament regeneration and graft-bone healing after reconstruction surgery using a silk-collagen scaffold. The scaffold was infiltrated by great many fibroblast-like cells and tenascin-C depositions at 4 and 16 weeks after surgery. The graft-bone interface exhibited good bone integration at 16 weeks after surgery. The results showed that BMSCs combined with silk-collagen scaffolds represent a good prospect in ACL tissue engineering and future clinical use.

Ideally, a tissue engineering scaffold for ACL reconstruction needs to simulate biological functions as well as the geometric structures of ligaments [32]. The ECM is important in guiding tissue ingrowth, maintaining homeostasis, and providing mechanical support during the ligament regeneration process. The silk-collagen scaffold takes advantage of silk's inherent mechanical properties and its suitability for knitting as well as the favorable biocompatibility of collagen matrix. Collagen matrix dominated the space between the silk fibroins and provided an attachment point for seeded cells.

The synovium layer covers the knee cavity, providing a less vascular microenvironment. At 4 and 16 weeks after surgery, fewer cells were attracted into the scaffold, and less ECM deposition occurred in the $S$ group compared to the $S / C$ group. According to the results, few cells migrated from surrounding tissues into the scaffold, and implanted BMSCs contributed proliferated cells to the scaffold and regenerated
ECM. Fan and colleagues found that the production of tenascin-C, collagen-II, and collagen-I from stem cells was greatly improved after cocultivation with silk scaffolds after 7 and 14 days [33]. Tenascin-C, one of the extracellular matrix glycoproteins in intra-articular grafts, is always expressed in the actively remodeling tissue and has a highly restricted gene expression model [34]. The tenascin-C expression in the $\mathrm{S}$ group was obviously lower than that in the S/C group at 4 and 16 weeks postoperatively. Results demonstrated that grafts in the S/C group exhibited more vibrant ligament regeneration than did those in the $S$ group, and implanted BMSCs contributed to cell proliferation and ECM deposition.

Successful ACL reconstruction requires solid graftbone healing [17]. Graft-bone healing in the bone tunnel requires that bone grow inward into the graft-bone interface. Kanaya and colleagues [35] reported that transected sections shrank over time, and the interface in the MSC(-) group still lacked tissue at all points in time postoperatively, whereas in the MSC(+) group, GFP-positive cells were found at 2 and 4 weeks postoperatively in healing tissues covering the transected section. The histologic score of the MSC(+) group was obviously better than that of the MSC(-) group. As reported by Hong and colleagues, BMSCs may promote the graft-bone healing process, as cartilage-like cells proliferated and perpendicular collagen fibers increasingly formed at 4 weeks postoperatively in a rabbit model [36]. In the present study, cartilage-like cells proliferated, and less fibrocartilage-like tissue formed at the graft-bone interface in the $\mathrm{S}$ group than in the S/C group at 4 weeks after surgery. The mature trabecular bone was found in the core part of the graft in the S/C group at 16 weeks postoperatively, whereas only the mature trabecular bone was found at the interface in the $S$ group. The graft-bone healing process may be promoted by the host stem cells from the circumambient bone marrow in the bone tunnel [37-39], but the host cell infiltration into the graft might take more than 4 weeks after surgery [20]. Based on the present study, it is considered that the implanted BMSCs mostly promoted bone integration between the graft and bone. 
Oka and colleagues found that bone integration at the graft-bone interface determined micro-CT parameters [40]. Micro-CT could discern subtle changes in bone tunnels and collect gross information of newly formed mineralized tissue through imaging [41]. This study evaluated bone integration using micro-CT. More mineralized tissues were detected in the bone tunnel in the $\mathrm{S} / \mathrm{C}$ group than in the $\mathrm{S}$ group at 4 and 16 weeks postoperatively; this finding corresponded to the histological findings.

The two main parameters of a ligament regenerated by tissue engineering are failure load and stiffness. Previous studies have shown that silk degraded via proteolytic degradation, resulting in a decrease in the scaffold mechanical strength $[42,43]$. The speed of decrease in mechanical strength depends primarily on the physiological status, mechanical environment, implantation site, and scaffold structure. ECM including collagen fibers and proteoglycans could be produced by the infiltrated cells, which makes up for the mechanical strength decrease due to degradation. The mean failure load and stiffness in the S/C group were greater than those in the $S$ group at 4 and 16 weeks after the procedure. The absence of a notable distinction between the two might be attributable to the small specimen dimensions.

One limitation of the present study was that we did not quantify the number of BMSCs implanted on the silk-collagen scaffold, and the optimal number of implanted cells remains unknown. Furthermore, the seeded BMSCs were not labeled and tracked, which represents another limitation. Although BMSCs seeded on the scaffold maintained good cellular morphology in vitro, the environments of the joint cavity and bone tunnel are different from that of a Petri dish. In a previous study, autologous BMSCs transfected with lentivirus vector expressing enhanced green fluorescent protein (Lv-eGFP) were seeded on a decellularized semitendinous tendon graft for ACL reconstruction [44]. The eGFPpositive cells could be observed at 12 weeks postoperatively; although, the eGFP-positive cell number at week 12 was significantly lower than that at week 4 . The conclusions of the present study were based on cell infiltration and tenascin-C deposition and on graft-bone healing observed histologically. More data will be needed to confirm the fate of the implanted cells in a future study.

\section{Conclusion}

BMSCs may promote ligament regeneration in the cavity and bone integration at the graft-bone interface. Silk-collagen scaffold and BMSCs are very likely to be combined for clinical practice in the future.

\section{Data Availability}

The data used to support the findings of this study are included within the article.

\section{Conflicts of Interest}

The authors declare that they have no conflicts of interest.

\section{Authors' Contributions}

Fanggang Bi conceived and designed the experiment. Fanggang $\mathrm{Bi}$, Yangdi Chen, Junqi Liu, and Wenhao Hu performed the experiment. Fanggang $\mathrm{Bi}$ and Yangdi Chen analyzed data. Ke Tian contributed reagents/materials/analysis tools. Fanggang Bi wrote the manuscript:. All authors read and approved the final manuscript.

\section{Acknowledgments}

This work was supported by the Foundation of Henan Educational Committee (19A320011) and the Key Project of Science and Technology Department of Henan Province-2020 (22170139).

\section{References}

[1] M. J. Price, M. Tuca, F. A. Cordasco, and D. W. Green, "Nonmodifiable risk factors for anterior cruciate ligament injury," Current Opinion in Pediatrics, vol. 29, no. 1, pp. 55-64, 2017.

[2] R. B. Frobell, E. M. Roos, H. P. Roos, J. Ranstam, and L. S. Lohmander, "A randomized trial of treatment for acute anterior cruciate ligament tears," The New England Journal of Medicine, vol. 363, no. 4, pp. 331-342, 2010.

[3] R. Vaishya, A. K. Agarwal, S. Ingole, and V. Vijay, "Current trends in anterior cruciate ligament reconstruction: a review," Cureus, vol. 7, no. 11, article e378, 2015.

[4] T. Diermeier, R. Tisherman, J. Hughes et al., "Quadriceps tendon anterior cruciate ligament reconstruction," Knee Surgery, Sports Traumatology, Arthroscopy, vol. 28, no. 8, pp. 26442656, 2020.

[5] S. Shumborski, L. J. Salmon, C. Monk, E. Heath, J. P. Roe, and L. A. Pinczewski, "Allograft donor characteristics significantly influence graft rupture after anterior cruciate ligament reconstruction in a young active population," The American Journal of Sports Medicine, vol. 48, no. 10, pp. 2401-2407, 2020.

[6] S. J. Tulloch, B. M. Devitt, C. J. Norsworthy, and C. Mow, "Synovitis following anterior cruciate ligament reconstruction using the LARS device," Knee Surgery, Sports Traumatology, Arthroscopy, vol. 27, no. 8, pp. 2592-2598, 2019.

[7] S. Rai, S. Y. Jin, B. Rai et al., "A single bundle anterior cruciate ligament reconstruction (ACL-R) using hamstring tendon autograft and tibialis anterior tendon allograft: a comparative study," Current Medical Science, vol. 38, no. 5, pp. 818-826, 2018.

[8] N. K. Paschos and S. M. Howell, "Anterior cruciate ligament reconstruction: principles of treatment," EFORT Open Reviews, vol. 1, no. 11, pp. 398-408, 2016.

[9] W. Shen, X. Chen, Y. Hu et al., "Long-term effects of knitted silk-collagen sponge scaffold on anterior cruciate ligament reconstruction and osteoarthritis prevention," Biomaterials, vol. 35, no. 28, pp. 8154-8163, 2014.

[10] C. T. Laurencin and J. W. Freeman, "Ligament tissue engineering: an evolutionary materials science approach," Biomaterials, vol. 26, no. 36, pp. 7530-7536, 2005.

[11] F. Bi, Z. Shi, A. Liu, P. Guo, and S. Yan, “Anterior cruciate ligament reconstruction in a rabbit model using silk-collagen scaffold and comparison with autograft," PLoS One, vol. 10, no. 5, article e0125900, 2015. 
[12] D. L. Barreiro, J. Yeo, A. Tarakanova, F. J. Martin-Martinez, and M. J. Buehler, "Multiscale modeling of silk and silkbased biomaterials-a review," Macromolecular Bioscience, vol. 19, no. 3, article e1800253, 2019.

[13] R. P. A. Janssen and S. U. Scheffler, "Intra-articular remodelling of hamstring tendon grafts after anterior cruciate ligament reconstruction," Knee Surgery, Sports Traumatology, Arthroscopy, vol. 22, no. 9, pp. 2102-2108, 2014.

[14] K. Ficek, J. Rajca, M. Stolarz et al., "Bioresorbable stent in anterior cruciate ligament reconstruction," Polymers, vol. 11, no. 12, p. 1961, 2019.

[15] D. Chanda, S. Kumar, and S. Ponnazhagan, "Therapeutic potential of adult bone marrow-derived mesenchymal stem cells in diseases of the skeleton," Journal of Cellular Biochemistry, vol. 111, no. 2, pp. 249-257, 2010.

[16] B. A. Tucker, S. S. Karamsadkar, W. S. Khan, and P. Pastides, "The role of bone marrow derived mesenchymal stem cells in sports injuries," Journal of Stem Cells, vol. 5, no. 4, pp. 155$166,2010$.

[17] C. Teng, C. Zhou, D. Xu, and F. Bi, "Combination of plateletrich plasma and bone marrow mesenchymal stem cells enhances tendon-bone healing in a rabbit model of anterior cruciate ligament reconstruction," Journal of Orthopaedic Surgery and Research, vol. 11, no. 1, p. 96, 2016.

[18] C. S. Linsley, B. M. Wu, and B. Tawil, "Mesenchymal stem cell growth on and mechanical properties of fibrin-based biomimetic bone scaffolds," Journal of Biomedical Materials Research. Part A, vol. 104, no. 12, pp. 2945-2953, 2016.

[19] T. Wang, X. Yang, X. Qi, and C. Jiang, "Osteoinduction and proliferation of bone-marrow stromal cells in threedimensional poly ( $\varepsilon$-caprolactone)/ hydroxyapatite/collagen scaffolds," Journal of Translational Medicine, vol. 13, no. 1, p. 152, 2015.

[20] H. Komiyama, Y. Arai, Y. Kajikawa et al., "The fate and role of bone graft-derived cells after autologous tendon and bone transplantation into the bone tunnel," Journal of Orthopaedic Science, vol. 18, no. 6, pp. 994-1004, 2013.

[21] M. Kobayashi, N. Watanabe, Y. Oshima, Y. Kajikawa, M. Kawata, and T. Kubo, "The fate of host and graft cells in early healing of bone tunnel after tendon graft," The American Journal of Sports Medicine, vol. 33, no. 12, pp. 1892-1897, 2005.

[22] J. K. Lim, J. Hui, L. Li, A. Thambyah, J. Goh, and E. H. Lee, "Enhancement of tendon graft osteointegration using mesenchymal stem cells in a rabbit model of anterior cruciate ligament reconstruction," Arthroscopy, vol. 20, no. 9, pp. 899910, 2004.

[23] M. Y. H. Soon, A. Hassan, J. H. P. Hui, J. C. H. Goh, and E. H. Lee, "An analysis of soft tissue allograft anterior cruciate ligament reconstruction in a rabbit model: a short-term study of the use of mesenchymal stem cells to enhance tendon osteointegration," The American Journal of Sports Medicine, vol. 35, no. 6, pp. 962-971, 2007.

[24] C. C. Ude, B. S. Shamsul, M. H. Ng et al., "Long-term evaluation of osteoarthritis sheep knee, treated with TGF- $\beta 3$ and BMP-6 induced multipotent stem cells," Experimental Gerontology, vol. 104, pp. 43-51, 2018.

[25] M. Chen, Y. Xu, T. Zhang et al., "Mesenchymal stem cell sheets: a new cell-based strategy for bone repair and regeneration," Biotechnology Letters, vol. 41, no. 3, pp. 305-318, 2019.
[26] W. Zhang, Y. Yang, K. Zhang, Y. Li, and G. Fang, "Weft-knitted silk-poly(lactide-co-glycolide) mesh scaffold combined with collagen matrix and seeded with mesenchymal stem cells for rabbit Achilles tendon repair," Connective Tissue Research, vol. 56, no. 1, pp. 25-34, 2015.

[27] W. Li, R. Xu, J. Huang, X. Bao, and B. Zhao, “Treatment of rabbit growth plate injuries with oriented ECM scaffold and autologous BMSCs," Scientific Reports, vol. 7, no. 1, article 44140, 2017.

[28] S. Q. Ruan, J. Deng, L. Yan, and W. L. Huang, "Composite scaffolds loaded with bone mesenchymal stem cells promote the repair of radial bone defects in rabbit model," Biomedicine \& Pharmacotherapy, vol. 97, pp. 600-606, 2018.

[29] J. S. Pieper, A. Oosterhof, P. J. Dijkstra, J. H. Veerkamp, and T. van Kuppevelt, "Preparation and characterization of porous crosslinked collagenous matrices containing bioavailable chondroitin sulphate," Biomaterials, vol. 20, no. 9, pp. $847-$ 858, 1999.

[30] M. L. Bouxsein, S. K. Boyd, B. A. Christiansen, R. E. Guldberg, K. J. Jepsen, and R. Müller, "Guidelines for assessment of bone microstructure in rodents using micro-computed tomography," Journal of Bone and Mineral Research, vol. 25, no. 7, pp. 1468-1486, 2010.

[31] A. J. Lee, W. H. Chung, D. H. Kim et al., “Anterior cruciate ligament reconstruction in a rabbit model using canine small intestinal submucosa and autologous platelet-rich plasma," The Journal of Surgical Research, vol. 178, no. 1, pp. 206-215, 2012.

[32] F. Wang, Y. Hu, D. He, G. Zhou, and E. Ellis III, "Scaffold-free cartilage cell sheet combined with bone-phase BMSCs-scaffold regenerate osteochondral construct in mini-pig model," American Journal of Translational Research, vol. 10, no. 10, pp. 2997-3010, 2018.

[33] H. Fan, H. Liu, E. J. W. Wong, S. L. Toh, and J. C. H. Goh, "In vivo study of anterior cruciate ligament regeneration using mesenchymal stem cells and silk scaffold," Biomaterials, vol. 29, no. 23, pp. 3324-3337, 2008.

[34] E. J. Mackie and S. Ramsey, "Expression of tenascin in jointassociated tissues during development and postnatal growth," Journal of Anatomy, vol. 188, pp. 157-165, 1996.

[35] A. Kanaya, M. Deie, N. Adachi, M. Nishimori, S. Yanada, and M. Ochi, "Intra-articular injection of mesenchymal stromal cells in partially torn anterior cruciate ligaments in a rat model," Arthroscopy, vol. 23, no. 6, pp. 610-617, 2007.

[36] H. W. Ouyang, J. C. H. Goh, and E. H. Lee, "Use of bone marrow stromal cells for tendon graft-to-bone healing: histological and immunohistochemical studies in a rabbit model," The American Journal of Sports Medicine, vol. 32, no. 2, pp. 321327, 2004.

[37] S. Kawamura, L. Ying, H. J. Kim, C. Dynybil, and S. A. Rodeo, "Macrophages accumulate in the early phase of tendon-bone healing," Journal of Orthopaedic Research, vol. 23, no. 6, pp. 1425-1432, 2005.

[38] M. Hevesi, M. LaPrade, D. B. F. Saris, and A. J. Krych, "Stem cell treatment for ligament repair and reconstruction," Current Reviews in Musculoskeletal Medicine, vol. 12, no. 4, pp. 446450, 2019.

[39] Z. C. Hao, S. Z. Wang, X. J. Zhang, and J. Lu, "Stem cell therapy: a promising biological strategy for tendon-bone healing after anterior cruciate ligament reconstruction," Cell Proliferation, vol. 49, no. 2, pp. 154-162, 2016. 
[40] S. Oka, T. Matsumoto, S. Kubo et al., "Local administration of low-dose simvastatin-conjugated gelatin hydrogel for tendonbone healing in anterior cruciate ligament reconstruction," Tissue Engineering. Part A, vol. 19, no. 9-10, pp. 1233-1243, 2013.

[41] M. Bellido, L. Lugo, J. A. Roman-Blas et al., "Improving subchondral bone integrity reduces progression of cartilage damage in experimental osteoarthritis preceded by osteoporosis," Osteoarthritis and Cartilage, vol. 19, no. 10, pp. 1228-1236, 2011.

[42] G. H. Altman, F. Diaz, C. Jakuba et al., "Silk-based biomaterials," Biomaterials, vol. 24, no. 3, pp. 401-416, 2003.

[43] D. Greenwald, S. Shumway, P. Albear, and L. Gottlieb, "Mechanical comparison of 10 suture materials before and after_in vivo_incubation," The Journal of Surgical Research, vol. 56, no. 4, pp. 372-377, 1994.

[44] W. Lu, J. Xu, S. Dong et al., “Anterior cruciate ligament reconstruction in a rabbit model using a decellularized allogenic semitendinous tendon combined with autologous bone marrow-derived mesenchymal stem cells," Stem Cells Translational Medicine, vol. 8, no. 9, pp. 971-982, 2019. 